\title{
A RESSONÂNCIA MAGNÉTICA DO QUADRIL NA AVALIAÇ̃̃o DE PACIENTES COM ARTRITE REUMATÓIDE: ESTUDO DESCRITIVO*
}

\author{
Wanda Chiyoko Iwakami Caldana ${ }^{1}$, Rozana Mesquita Ciconelli ${ }^{2}$, Yara Juliano ${ }^{3}$, Artur da Rocha \\ Corrêa Fernandes ${ }^{4}$
}

Resumo OBJETIVO: Avaliar o valor da ressonância magnética (RM) como modalidade diagnóstica de imagem em pacientes com artrite reumatóide e estimar a freqüência das alterações no quadril em pacientes sintomáticos e assintomáticos. MATERIAIS E MÉTODOS: Foram avaliados 40 pacientes 17 sintomáticos e 23 assintomáticos) em equipamento de RM com 1,5 T, com o uso do contraste endovenoso. Os achados de RM foram subdivididos em alterações inflamatórias, degenerativas, mistas (inflamatórias e degenerativas) e extraarticulares. RESULTADOS: As alterações mais freqüentes na RM de quadril dos pacientes sintomáticos e assintomáticos foram dos tipos degenerativas (22,5\%) e extra-articulares $(22,5 \%)$. Apenas cinco dos pacientes sintomáticos apresentaram achados inflamatórios $(12,5 \%)$, sendo quatro $(10 \%)$ com alterações degenerativas associadas (alterações mistas). CONCLUSÃO: 0 estudo permitiu avaliar as principais alterações encontradas em pacientes portadores de artrite reumatóide pela RM. As alterações mais freqüentes na RM do quadril desses pacientes foram dos tipos degenerativas e extra-articulares $(22,5 \%$ de cada grupo). Apenas cinco dos pacientes sintomáticos apresentaram achados inflamatórios $(12,5 \%)$, sendo quatro $(10 \%)$ com alterações degenerativas associadas.

Unitermos: Ressonância magnética; Quadril; Artrite reumatóide; Adulto.

Abstract Magnetic resonance imaging of the hip in rheumatoid arthritis patients: a descriptive study.

OBJECTIVE: To assess the usefulness of MRI as a diagnostic method in patients with rheumatoid arthritis, and to evaluate the frequency of changes in the hip in symptomatic and asymptomatic patients. MATERIALS AND METHODS: Forty patients (17 symptomatic and 23 asymptomatic) were evaluated by $1.5 \mathrm{~T}$ magnetic resonance imaging (MRI) after intravenous contrast media injection. MRI findings were divided in inflammatory, degenerative, combined (inflammatory and degenerative) and extra-articular changes. RESULTS: The most frequent findings seen were degenerative and extra-articular changes $(22.5 \%$ each). Inflammatory changes were seen in only five $(12.5 \%)$ symptomatic patients, four of them with degenerative findings associated $(10 \%)$. CONCLUSION: The use of MRI of the hip allowed the evaluation of the main articular abnormalities seen in patients with rheumatoid arthritis. The most frequent findings were degenerative and extra-articular changes. Inflammatory findings were seen in only five patients, four of them with degenerative changes associated.

Key words: Magnetic resonance imaging; Hip; Rheumatoid arthritis; Adult.

\section{INTRODUÇÃO}

A artrite reumatóide (AR) é uma doença inflamatória crônica de acometimento sistêmico que promove destruição e limitação funcional das articulações sinoviais, podendo estar associada a manifestações

* Trabalho realizado no Departamento de Diagnóstico por Imagem da Escola Paulista de Medicina/Universidade Federal de São Paulo (EPM/Unifesp), São Paulo, SP.

1. Pós-graduanda do Departamento de Diagnóstico por Imagem da EPM/Unifesp, Mestrado pelo Departamento de Diagnóstico por Imagem da EPM/Unifesp.

2. Docente da Disciplina de Reumatologia da EPM/Unifesp.

3. Docente do Departamento de Epidemiologia e Bioestatís tica da EPM/Unifesp.

4. Docente do Departamento de Diagnóstico por Imagem da EPM/Unifesp.

Endereço para correspondência: Dra. Wanda Chiyoko Iwakami Caldana. Rua Joaquim Távora, 1299, ap. 82, Vila Mariana. São Paulo, SP, 04015-002. E-mail: wiwakami@ig.com.br.

Recebido para publicação em 24/6/2004. Aceito, após revisão, em 14/12/2004. extra-articulares como nódulos reumatóides, alterações oculares e pulmonares, vasculite e pericardite ${ }^{(1)}$. Sua prevalência mundial é de $0,5 \%$ a $1 \%$ da população ${ }^{(1-4)}$, sendo estimada, no Brasil, entre $0,2 \%$ e $1,0 \%{ }^{(5)}$.

A AR acomete adultos entre a quarta e sexta décadas de vida, economicamente ativos, sendo as mulheres duas a três vezes mais afetadas que os homens ${ }^{(\mathbf{1 , 2}, \mathbf{4})}$. Seu caráter destrutivo leva a perda progressiva da função articular e redução da qualidade de vida dos pacientes ${ }^{(\mathbf{1})}$.

Apesar de sua etiologia ainda não estar bem esclarecida, há estudos enfocando a participação de fatores genéticos e possíveis agentes infecciosos que atuariam no mecanismo da perpetuação da resposta inflamatória, podendo explicar o desenvolvimento desta $\operatorname{artropatia}^{(\mathbf{2 , 5})}$.
A AR é caracterizada pela inflamação e proliferação da sinóvia, que promove destruição tanto da cartilagem e osso subcondral, com conseqüente perda da função articular, como das estruturas extraarticulares envolvidas pela membrana sinovial - bolsas e tendões ${ }^{(\mathbf{2 , 6})}$. Geralmente compromete várias articulações de forma simétrica e bilateral, predominando nas mãos, punhos, pés e joelhos ${ }^{(2)}$, avançando para outras articulações com a progressão da doença ${ }^{(7)}$. As articulações periféricas são freqüentemente acometidas no primeiro ano da AR, nas quais a atividade da doença pode ser facilmente caracterizada pelo exame físico, palpando-se articulações com calor, edema e dor, com representação radiográfica caracterizada pelo aumento de partes moles, osteopenia 
periarticular e erosões ósseas ${ }^{(7)}$. A literatura mostra poucos dados sobre a frequiência e o período de acometimento das grandes articulações como quadril, ombro, joelho, cotovelo e tornozelo. Alguns estudos mostram que estas articulações são acometidas numa fase tardia da $\mathrm{AR}^{(7)}$; outros estudos afirmam que tanto as pequenas quanto as grandes articulações podem estar envolvidas já na fase inicial da doença ${ }^{(\mathbf{8})}$.

Ao contrário das articulações superficiais de mãos e pés, o quadril não é facilmente acessível pela avaliação clínica, limitando a caracterização de seu eventual envolvimento, geralmente por sinovite ou derrame $\operatorname{articular}^{(\mathbf{8})}$. Além disso, muitos pacientes podem não ter nenhum sintoma no início da doença, retardando a abordagem diagnóstica dessa articulação somente para o momento em que surgir alguma queixa clínica, quando alterações mais avançadas já se instalaram ${ }^{(7,8)}$.

Por ser uma articulação de carga, o quadril pode também apresentar dor decorrente de alterações degenerativas, tanto pela idade quanto secundárias aos processos reumatológicos, limitando seu correto diagnóstico sem o auxílio de uma propedêutica de imagem ${ }^{(9)}$. Além disso, nesses pacientes é importante afastar a possibilidade de osteonecrose, potencial complicação da corticoterapia prolongada ${ }^{(\mathbf{1 0})}$.

$\mathrm{O}$ restrito conhecimento sobre as alterações promovidas pela AR no quadril e a dificuldade da abordagem clínica desta articulação motivaram o interesse em estudá-la pela ressonância magnética (RM), excelente método de imagem na avaliação do espaço articular, da medular óssea e das partes moles periarticulares. Talvez a maior vantagem desta técnica esteja na detecção da atividade da doença pela pesquisa do espessamento e realce da membrana sinovial (sinovite), facilmente demonstrada pela injeção endovenosa do contraste paramagnético (gadolínio) ${ }^{(6,11-14)}$. A RM destaca-se ainda por ser método não invasivo, útil como complemento da avaliação clínica articular, não só para detecção das alterações iniciais da doença, mas também no controle evolutivo da AR, monitoramento do tratamento e diagnóstico diferencial com outras doenças ${ }^{(\mathbf{2 , 6}, 15-18)}$.

Considerando-se os escassos conhecimentos sobre o envolvimento do quadril pela AR e o potencial da RM na avaliação articular, idealizamos este estudo com os objetivos de pesquisar a frequiência das alterações na articulação do quadril em pacientes portadores de AR e avaliar o valor da RM como modalidade diagnóstica de imagem em pacientes com AR.

\section{MATERIAIS E MÉTODOS}

No período de abril de 2001 a dezembro de 2002, foi estudada a articulação do quadril de 40 pacientes com AR, de procedência ambulatorial, por meio da RM com gadolínio endovenoso. Na ausência de queixas específicas do quadril, convencionamos realizar o estudo da articulação esquerda. Na presença de alguma queixa, foi estudada a articulação sintomática.

Os pacientes foram subdivididos em três grupos, de acordo com o tempo de doença (< 2 anos; de 2 a 5 anos; $>5$ anos) e segundo a classe funcional (I a IV) estabelecida pelo American College of Rheumatology (ACR) em 1991 ${ }^{(19)}$.

Os critérios de inclusão adotados no estudo foram: pacientes com idade superior a 18 anos, conscientes e colaborativos, com diagnóstico de AR de acordo com os critérios estabelecidos na revisão pelo ACR em 1987(20). Estabelecemos como critérios de exclusão pacientes com diagnóstico de AR juvenil, quadril com próteses ou hastes, anquilose na articulação a ser estudada, contra-indicações absolutas ao exame de RM (marcapasso cardíaco e clipes de aneurismas cerebrais) e claustrofobia. Nos pacientes assintomáticos, com prótese ou anquilose da articulação esquerda, procedemos à avaliação do lado direito.

Os exames foram realizados em equipamento de RM marca Philips, modelo ACS/ NT, operando em alto campo magnético (1,5 T). Foi utilizada bobina de corpo e de superfície (circular - C1) para transmissão e recepção de sinais.

$\mathrm{O}$ exame do quadril foi realizado com a técnica TSE nos planos transversal e sagital, com seqüências ponderadas em T1, TSE transversal ponderada em T2 e STIR coronal ponderada em T2. Foram obtidas também sequiências SPIR coronal e transversal em T1 após injeção por via endovenosa do gadolínio (Gd-DTPA) em volume de $0,2 \mathrm{ml} / \mathrm{kg}$. A área para aquisição dos dados no plano coronal foi planejada paralela ao colo femoral, no plano sagital perpendicular ao colo e no plano transversal perpendicular ao corpo do paciente.

Os achados na RM foram agrupados em quatro categorias:

Alterações inflamatórias: Presença de espessamento sinovial maior que $2 \mathrm{~mm}$ de espessura com realce após o contraste, redução difusa ou medial do espaço articular/afilamento condral e subcondral, edema da medular óssea, alterações císticas subcondrais, derrame articular, bursite trocantérica e/ou do iliopsoas

Alterações degenerativas: Redução do espaço articular superior, superior intermediário ou súpero-medial, afilamento condral, esclerose subcondral, alterações císticas subcondrais, osteófitos.

Alterações mistas: Alterações inflamatórias e degenerativas.

Achados extra-articulares: Tendinopatia dos glúteos mínimo e ou médio, acometimento das bolsas do iliopsoas, trocantérica ou dos glúteos.

Para análise dos resultados foram aplicados o teste do qui-quadrado $\left(\chi^{2}\right)$ ou teste exato de Fisher para avaliar possíveis associações dos pacientes sintomáticos e assintomáticos em relação às variáveis estudadas e o teste de Mann-Whitney para comparar o tempo de doença entre pacientes sintomáticos e assintomáticos. $\mathrm{O}$ nível de rejeição da hipótese de nulidade foi fixado em 0,05 ou $5 \%(\alpha \leq 0,05)$.

\section{RESULTADOS}

Os 40 pacientes estudados foram constituídos por seis homens e 34 mulheres, com idade entre 29 e 70 anos (mediana de 53 anos, média de 51,7 anos), sendo 17 sintomáticos e 23 assintomáticos. Dentre eles, a maioria pertencia aos grupos funcionais I e II (sete indivíduos em cada grupo).

Utilizando o teste de Mann-Whitney, observamos que pacientes sintomáticos apresentavam mais tempo de doença (acima de cinco anos) em relação aos pacientes assintomáticos $(\mathrm{p}<0,035)$.

No grupo dos sintomáticos, 15 apresentaram exames de RM alterados, e no grupo dos assintomáticos, 11 apresentaram alterações na $\mathrm{RM}$ (Tabela 1 ). 
Na Tabela 2 notamos que a distribuição dos achados mostrou predomínio das alterações degenerativas e extra-articulares, envolvendo $22,5 \%$ dos pacientes estudados em cada um destes grupos (Figuras 1 a 4). No grupo dos sintomáticos tais alterações corresponderam, respectivamente, a $35,3 \%$ e $23,5 \%$.

Nas Tabelas 2 e 3 observamos sinais de artropatia inflamatória em cinco pacientes do grupo dos sintomáticos, que apresentaram sinovite, redução do espaço articular/ erosão condral, erosões subcondrais e edema da medular óssea (Figuras 5 e 6). Dentre estes, quatro também demonstraram alterações degenerativas associadas (Tabela 4 e Figura 7). Todos os pacientes com sinovite apresentaram espessamento sinovial maior que $2 \mathrm{~mm}$ (Tabela 5).

No grupo dos assintomáticos encontramos três pacientes com espessura da membrana sinovial menor que $2 \mathrm{~mm}$, porém com realce após o uso do contraste endovenoso (Tabela 2).

\section{DISCUSSÃO}

Os pacientes sintomáticos e assintomáticos estudados na nossa amostra foram na maioria do sexo feminino acima de 35 anos e pertencentes aos grupos funcionais I e II. Os pacientes com sintomas no quadril apresentaram maior tempo de doença (acima de cinco anos) e alterações no exame de RM (alterações mistas e espessura da membrana sinovial maior que $2 \mathrm{~mm}$ ) quando comparados aos pacientes assintomáticos, concordante com a literatura ${ }^{(21)}$.

Avaliando os achados na RM dos 17 pacientes sintomáticos, somente cinco apresentaram atividades inflamatórias da doença, sendo quatro associados a alterações degenerativas. A maioria apresentou alterações degenerativas e extra-articulares. A freqüência da osteoartrose nos pacientes estudados pode ser atribuída à faixa etária dos indivíduos, com maior suscetibilidade às alterações degenerativas, concordante com a literatura ${ }^{(\mathbf{9})}$.

Dos 23 pacientes assintomáticos, cinco $(21,7 \%)$ apresentavam sinais de alterações extra-articulares, três mostravam alterações degenerativas $(13 \%)$ e em três havia realce sinovial com espessura menor que 2 mm (13\%) (Tabela 2). Nenhum destes

Tabela 1 Pacientes sintomáticos ou assintomáticos com exame de ressonância magnética normal e alterado.

\begin{tabular}{|l|c|c|c|c|}
\hline $\begin{array}{l}\text { Exame de ressonância } \\
\text { magnética }\end{array}$ & Sintomáticos & Assintomáticos & Total & $\begin{array}{c}\text { Porcentagem } \\
\text { de sintomáticos }\end{array}$ \\
\hline Normal & 2 & 12 & 14 & $14,3 \%$ \\
Alterado & 15 & 11 & 26 & $57,7 \%$ \\
\hline Total & 17 & 23 & 40 & $42,5 \%$ \\
\hline
\end{tabular}

Teste do qui-quadrado $\left(p<0,01^{*}\right)$.

Tabela 2 Distribuição dos achados de ressonância magnética nos pacientes sintomáticos ou assintomáticos.

\begin{tabular}{|c|c|c|c|c|c|c|}
\hline \multirow[b]{2}{*}{ Achados na ressonância magnética } & \multicolumn{2}{|c|}{ Sintomáticos } & \multicolumn{2}{|c|}{ Assintomáticos } & \multirow[b]{2}{*}{ Total } & \multirow[b]{2}{*}{ Porcentagem } \\
\hline & $\mathrm{N}$ & $\%$ & $\mathrm{~N}$ & $\%$ & & \\
\hline Normal & 2 & 11,8 & 12 & 52,2 & 14 & $35,0 \%$ \\
\hline Alterações degenerativas & 6 & 35,3 & 3 & 13,0 & 9 & $22,5 \%$ \\
\hline Alterações inflamatórias* & 1 & 5,9 & 0 & 0,0 & 1 & $2,5 \%$ \\
\hline Alterações mistas* & 4 & 23,5 & 0 & 0,0 & 4 & $10,0 \%$ \\
\hline Alterações extra-articulares & 4 & 23,5 & 5 & 21,7 & 9 & $22,5 \%$ \\
\hline Membrana sinovial $<2 \mathrm{~mm}$ e realce & 0 & 0,0 & 3 & 13,0 & 3 & $7,5 \%$ \\
\hline Total & 17 & 100,0 & 23 & 100,0 & 40 & $100,0 \%$ \\
\hline
\end{tabular}

* Membrana sinovial maior que $2 \mathrm{~mm}$ de espessura.

Tabela 3 Pacientes sintomáticos ou assintomáticos com alterações inflamatórias.

\begin{tabular}{|l|c|c|c|c|}
\hline \multirow{2}{*}{ Pacientes } & \multicolumn{3}{|c|}{ Alterações inflamatórias } & \multirow{2}{*}{ Porcentagem (presente) } \\
\cline { 2 - 4 } & Presente & Ausente & Total & $5,9 \%$ \\
Sintomáticos & 1 & 16 & 17 & $0,0 \%$ \\
Assintomáticos & 0 & 23 & 23 & $2,5 \%$ \\
\hline Total & 1 & 40 & 40 & P0 \\
\hline
\end{tabular}

Teste exato de Fisher, $p=0,42$ (não significante).

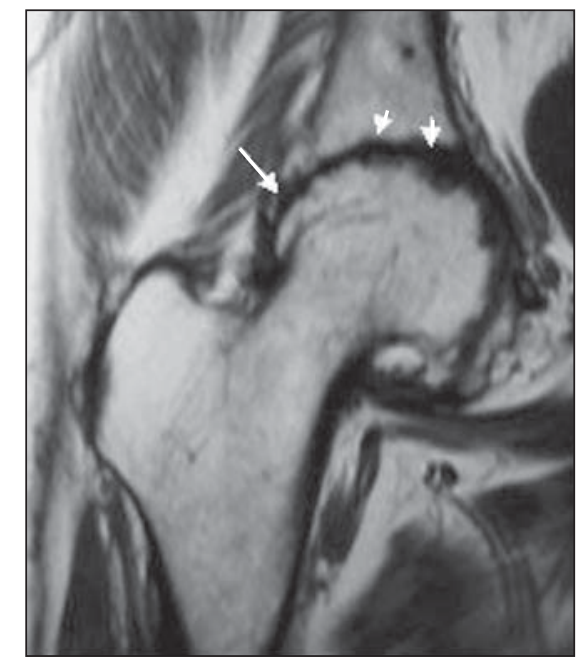

Figura 1. Alterações degenerativas: osteófitos marginais (seta), esclerose subcondral e afilamento condral difuso (cabeças de setas). RM plano coronal TSE ponderada em T1.

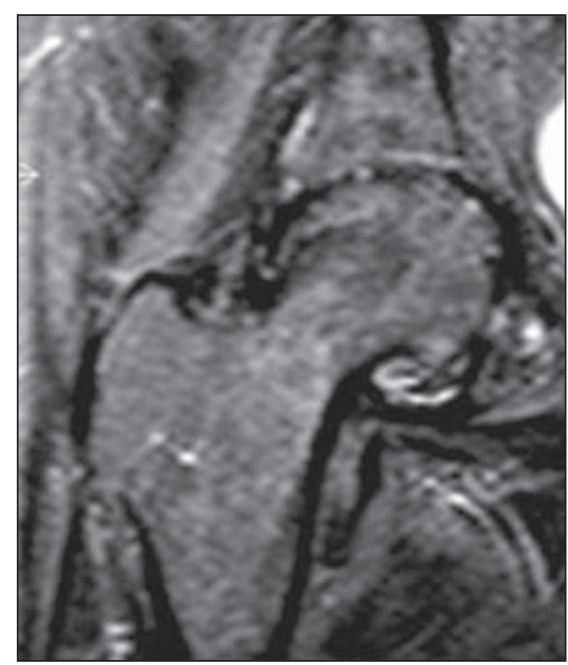

Figura 2. Alterações degenerativas. RM plano coronal STIR ponderada em T1: sem espessamento sinovial. 


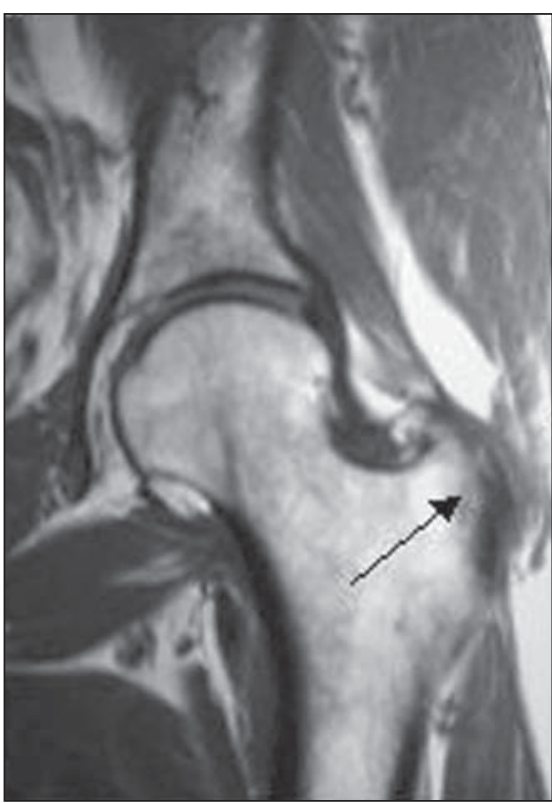

Figura 3. Alterações extra-articulares: espessamento do tendão glúteo mínimo junto ao trocanter maior (seta). RM plano coronal TSE ponderada em T2.

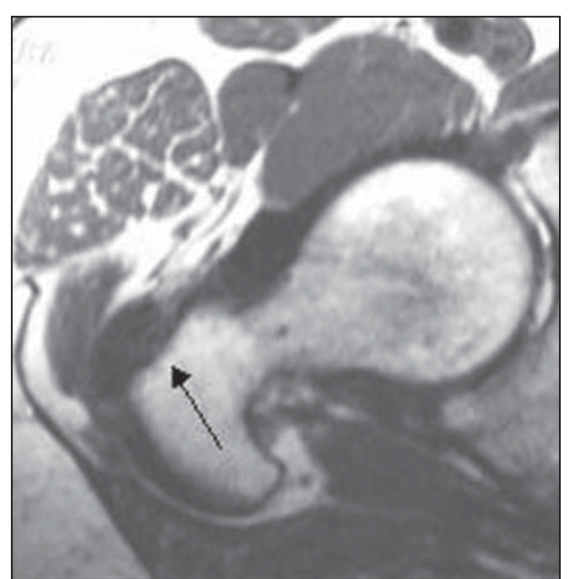

Figura 4. Alterações extra-articulares: espessamento do tendão glúteo mínimo junto ao trocanter maior (seta). RM plano transversal TSE ponderada em T1.

Tabela 4 Pacientes sintomáticos ou assintomáticos com alterações mistas.

\begin{tabular}{|l|c|c|c|c|}
\hline \multirow{2}{*}{ Pacientes } & \multicolumn{3}{|c|}{ Alterações mistas } & \multirow{2}{*}{ Porcentagem (presentes) } \\
\cline { 2 - 4 } & Presentes & Ausentes & Total & $23,5 \%$ \\
\hline Sintomáticos & 4 & 13 & 17 & $0,0 \%$ \\
Assintomáticos & 0 & 23 & 23 & $10,0 \%$ \\
\hline Total & 4 & 36 & 40 & \multirow{2}{*}{. } \\
\hline
\end{tabular}

Teste exato de Fisher, $\mathrm{p}=0,02^{*}$.

Tabela 5 Pacientes sintomáticos ou assintomáticos com espessamento da membrana sinovial maior que $2 \mathrm{~mm}$.

\begin{tabular}{|l|c|c|c|c|}
\hline \multirow{2}{*}{ Pacientes } & \multicolumn{2}{|c|}{ Espessamento sinovial maior que 2 mm } & \multirow{2}{*}{ Porcentagem (presente) } \\
\cline { 2 - 4 } & Presente & Ausente & Total & $29,4 \%$ \\
\hline Sintomáticos & 5 & 12 & 17 & $0,0 \%$ \\
Assintomáticos & 0 & 23 & 23 & $12,5 \%$ \\
\hline Total & 5 & 35 & 40 & \multirow{2}{*}{ (23) } \\
\hline
\end{tabular}

Teste exato de Fisher, $\mathrm{p}=0,009 *$.

pacientes apresentou alterações inflamatórias evidentes relacionadas à $\mathrm{AR}$, mostrando que a tendência da RM ser negativa nos pacientes assintomáticos em relação à atividade da doença é maior quando comparada ao grupo dos sintomáticos. Estes dados tendem a mostrar que a RM no quadril pode ser útil somente na avaliação de pacientes com algum sintoma e não como método de rastreamento.
Nos 40 pacientes avaliados, encontramos três indivíduos assintomáticos que apresentaram realce da membrana sinovial com espessura menor que $2 \mathrm{~mm}$. A grande questão está na valorização deste achado como patológico ou não. Na literatura existem poucos trabalhos avaliando o realce da membrana sinovial em indivíduos normais ${ }^{(22-29)}$. Todos estudaram a articulação do joelho e obtiveram resultados heterogê-

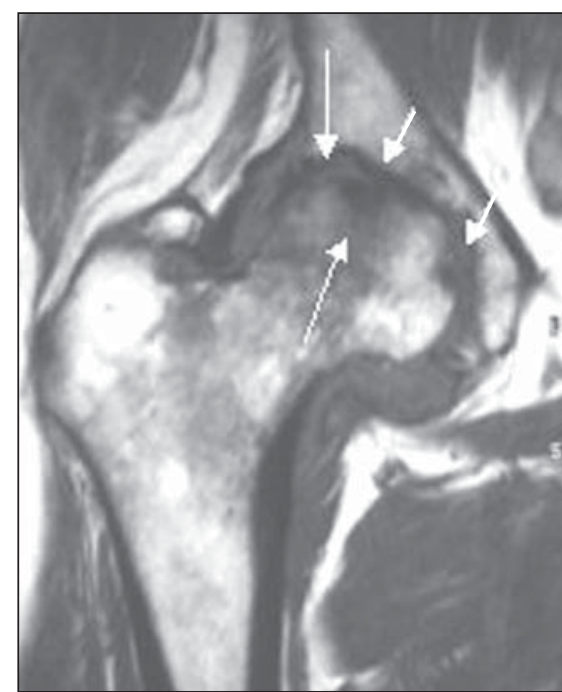

Figura 5. Alterações inflamatórias: afilamento/erosão condral (setas menores), erosão subcondral (setas maiores) RM plano coronal TSE ponderada em T1.

neos, alguns mostrando que a membrana sinovial normal não realça ${ }^{(22-26)} \mathrm{e}$ outros evidenciando o realce ${ }^{(28-30)}$. Em comum, todos esses trabalhos descreveram amostras reduzidas (inferior a dez indivíduos). Sabe-se ainda que pode ocorrer realce sinovial após atividade física, o que poderia explicar alguns dos casos de realce em indivíduos normais ${ }^{(31,32)}$. Por isso, para estabelecer se a sinovite é importante, deve-se não somente caracterizar o realce, mas também o espessamento associado da membrana, o que fizemos com o adequado cuidado.

Como citado acima, a movimentação ativa e passiva da articulação aumenta a permeabilidade vascular sinovial, a pressão plasmática e o gradiente de pressão, acelerando a passagem do líquido sinovial para o espaço articular ${ }^{(31,32)}$. Este princípio é usado nos estudos por artrografia indireta pela RM, em que, após a injeção do meio de contraste endovenoso e movimentação ativa da articulação em estudo, obtêm-se imagens com líquido articular contendo o contraste paramagnético que atravessa rapidamente a membrana sinovial, chegando no espaço articular ${ }^{(31,33,34)}$. Em nossos pacientes, uma simples caminhada até o local do exame poderia aumentar a permeabilidade vascular no quadril, mesmo antes 


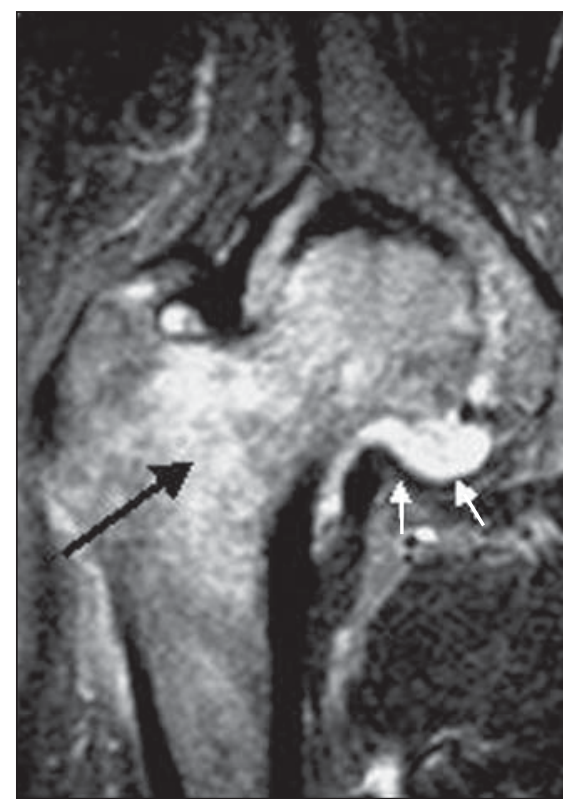

Figura 6. Alterações inflamatórias: edema da medular óssea (seta preta) e sinovite (setas brancas). RM plano coronal STIR ponderada em T2.

da injeção endovenosa do contraste, o que poderia justificar o achado de realce da membrana sinovial sem espessamento em três pacientes (Tabela 2).

Uma outra explicação para esta alteração sinovial seria a correlação com a fase angiogênica do processo inflamatório na $\mathrm{AR}$, em que, histologicamente, há uma hiperplasia da membrana sinovial com proliferação dos linfócitos perivasculares, do tecido necrótico e proliferação das células $\mathrm{T}$ e B, sem promover sintomas ao paciente, um estágio que precederia a proliferação sinovial propriamente dita, em que o paciente já apresenta quadro clínico de dor, edema e rigidez matinal, além da caracterização da sinovite pela $\mathrm{RM}$, ultrasonografia ou mesmo a cintilografia ${ }^{(35)}$. A maioria dos estudos pela RM caracteriza a sinovite associada ao espessamento da membrana sinovial que realça após o contraste. Nos três indivíduos da nossa amostra, a espessura da membrana foi menor que $2 \mathrm{~mm}$, podendo-se considerar como normal, quando comparada aos outros pacientes sintomáticos que apresentaram sinovite e espessamento maior que $2 \mathrm{~mm}$. A melhor forma de elucidar o significado do realce sinovial desses três pacientes será em reavaliá-los no futuro.

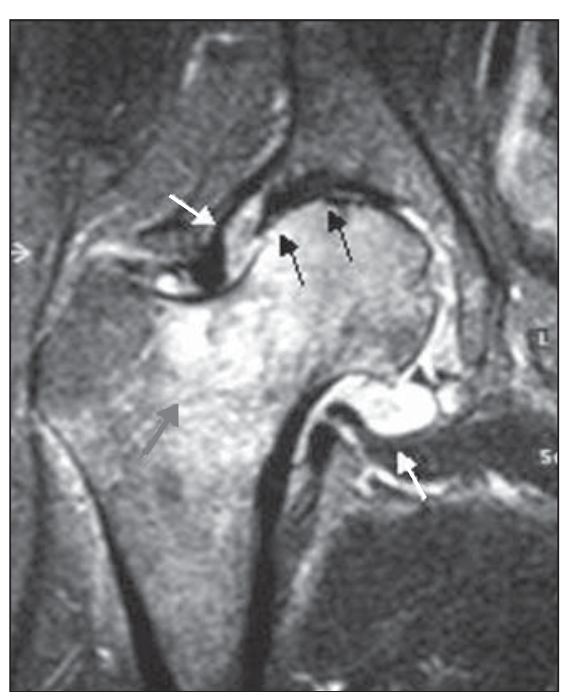

Figura 7. Alterações mistas: sinovite/pannus (setas brancas), edema de medular óssea (seta cinza), redução do espaço articular superior com esclerose subcondral e osteófitos marginais (setas pretas). RM plano coronal STIR ponderada em T2.

$\mathrm{Na}$ interpretação dos dados, tentamos estabelecer relação entre os achados extraarticulares com outros sinais indicativos do envolvimento articular pela AR. Não houve correlação na freqüência desses achados no grupo de pacientes estudados: a tendinopatia dos músculos glúteos junto à inserção trocantérica, por exemplo, foi encontrada em quatro pacientes sintomáticos $(23,5 \%)$ e em cinco assintomáticos $(21,7 \%)$, correspondendo a nove pacientes $(22,5 \%$ da amostra), todos sem alterações intra-articulares inflamatórias (como sinovite, erosões condrais e subcondrais).

As alterações extra-articulares podem ser decorrentes de processos patológicos ou desvios funcionais (como alterações de postura ou hipersolicitação), que podem ou não ser agravadas ou aceleradas pelas alterações de natureza reumatológica. A articulação do quadril pode sofrer mudanças na sua arquitetura habitual decorrentes de fatores intrínsecos e extrínsecos. A própria marcha pode se alterar por problemas nas articulações do joelho ou tornozelo, sobrecarregando um grupamento muscular ou mesmo causando atrito das estruturas por hipersolicitação ${ }^{(36)}$. Portanto, a correlação da frequiência destes achados com a doença reumática ainda está por ser comprovada, mas a tendência indicada por nossos resultados é atribuir os achados extra-articulares apenas às alterações de natureza biomecânica.

\section{CONCLUSÕES}

O estudo da articulação do quadril pela RM permitiu caracterizar as principais alterações encontradas em pacientes com AR. As alterações mais frequientes na RM do quadril dos pacientes, tanto sintomáticos como assintomáticos, foram dos tipos degenerativas $(22,5 \%)$ e extra-articulares $(22,5 \%)$. Apenas cinco dos pacientes sintomáticos apresentaram achados inflamatórios $(12,5 \%)$, sendo quatro $(10 \%)$ com alterações degenerativas associadas.

\section{REFERÊNCIAS}

1. Smolen JS, Steiner G. Therapeutic strategies for rheumatoid arthritis. Nat Rev Drug Discov 2003 2:473-88.

2. Scutellari PN, Orzincolo C. Rheumatoid arthritis: sequences. Eur J Radiol 1998;27 Suppl 1:S31-8.

3. Fex E, Jonsson K, Johnson U, Eberhardt K. Development of radiographic damage during the first 5 $6 \mathrm{yr}$ of rheumatoid arthritis. A prospective follow up study of a Swedish cohort. Br J Rheumatol 1996;35:1106-15.

4. Alarcon GS. Epidemiology of rheumatoid arthritis Rheum Dis Clin North Am 1995;21:589-604.

5. Brandão L, Sato EI, Ferraz MB, Andrade LEC. Etiopatogenia da artrite reumatóide. Rev Bras Reumatol 1994;34:93-9.

6. Winalski CS, Palmer WE, Rosenthal DI, Weissman BN. Magnetic resonance imaging of rheumatoid arthritis. Radiol Clin North Am 1996;34:243-58, X.

7. Kuper HH, van Leeuwen MA, van Riel PL, et al. Radiographic damage in large joints in early rheumatoid arthritis: relationship with radiographic damage in hands and feet, disease activity, and physical disability. Br J Rheumatol 1997;36:85560.

8. Eberhardt K, Fex E, Johnsson K, Geborek P. Hip involvement in early rheumatoid arthritis. Ann Rheum Dis 1995;54:45-8.

9. Drossaers-Bakker KW, Kroon HM, Zwinderman AH, Breedveld FC, Hazes JM. Radiographic damage of large joints in long-term rheumatoid arthritis and its relation to function. Rheumatology $(\mathrm{Ox}-$ ford) 2000;39:998-1003.

10. Resnick D, Niwayama G. Diagnosis of bone and joint disorders. 2nd ed. Philadelphia: Saunders, 1988.

11. Kainberger F, Trattnig S, Czerny C, Seidl G, Kritz $\mathrm{H}$, Imhof $\mathrm{H}$. MRI in assessment of the systemic manifestations of rheumatological disease. $\mathrm{Br} \mathrm{J}$ Rheumatol 1996;35 Suppl 3:40-4.

12. Cervilla VC. Resonancia magnética en reumatología. Reumatología 1997;13:137-46.

13. Lamer S, Sebag GH. MRI and ultrasound in children with juvenile chronic arthritis. Eur J Radiol 2000;33:85-93.

14. Abreu Júnior L, Vilela SA, Natour J, Fernandes ARC. Ressonância magnética (RM): contribuição para o diagnóstico e avaliação do acometimento da 
coluna cervical pela artrite reumatóide. Rev Bras Reumatol 1995;35:282-4.

15. Waterton JC, Rajanayagam V, Ross BD, Brown D, Whittemore A, Johnstone D. Magnetic resonance methods for measurement of disease progression in rheumatoid arthritis. Magn Reson Imaging 1993; 11:1033-8.

16. Sella EMC, Fernandes ARC, Ciconelli RC, Sato EI Ressonância magnética nas artropatias inflamatórias. Rev Bras Reumatol 1998;28:269-74.

17. Creamer P, Keen M, Zananiri F, et al. Quantitative magnetic resonance imaging of the knee: a method of measuring response to intra-articular treatments. Ann Rheum Dis 1997;56:378-81.

18. Huang J, Stewart N, Crabbe J, et al. A 1-year follow-up study of dynamic magnetic resonance imaging in early rheumatoid arthritis reveals synovitis to be increased in shared epitope-positive patients and predictive of erosions at 1 year. Rheumatology (Oxford) 2000;39:407-16.

19. Hochberg MC, Chang RW, Dwosh I, Lindsey S, Pincus T, Wolfe F. The American College of Rheumatology 1991 revised criteria for the classification of global functional status in rheumatoid arthritis. Arthritis Rheum 1992;35:498-502.

20. Arnett FC, Edworthy SM, Bloch DA, et al. The American Rheumatism Association 1987 revised criteria for the classification of rheumatoid arthritis. Arthritis Rheum 1988;31:315-24.

21. Lehtimaki MY, Kautiainen H, Hamalainen MM, et al. Hip involvement in seropositive rheumatoid ar- thritis. Survivorship analysis with a 15-year followup. Scand J Rheumatol 1998;27:406-9.

22. Bjorkengren AG, Geborek P, Rydholm U, Holtas S, Petterson H. MR imaging of the knee in acute rheumatoid arthritis: synovial uptake of gadoliniumDOTA. AJR 1990;155:329-32.

23. Kursunoglu-Brahme S, Riccio T, Weisman MH, et al. Rheumatoid knee: role of gadopentetate-enhanced MR imaging. Radiology 1990;176:831-5.

24. Oliver C, Speake S, Watt I, Dieppe P, Ratcliffe G. Advantages of an increased dose of MRI contrast agent for enhancing inflammatory synovium. Clin Radiol 1996;51:487-93.

25. Whitten CG, Moore TE, Yuh WT, Kathol MH, Renfrew DL, Walker CW. The use of intravenous gadopentetate dimeglumine in magnetic resonance imaging of synovial lesions. Skeletal Radiol 1992; 21:215-8.

26. Konig H, Sieper J, Wolf KJ. Rheumatoid arthritis: evaluation of hypervascular and fibrous pannus with dynamic MR imaging enhanced with GdDTPA. Radiology 1990;176:473-7.

27. Ostergaard M, Stoltenberg M, Henriksen O, Lorenzen I. The accuracy of MRI-determined synovial membrane and joint effusion volumes in arthritis. A comparison of pre- and post-aspiration volumes. Scand J Rheumatol 1995;24:305-11.

28. Ostergaard M, Lorenzen I, Henriksen O. Dynamic gadolinium-enhanced MR imaging in active and inactive immunoinflammatory gonarthritis. Acta Radiol 1994;35:275-81
29. Boegard T, Johansson A, Rudling O, Petersson I, Forslind K, Jonsson K. Gadolinium-DTPA-enhanced MR imaging in asymptomatic knees. Acta Radiol 1996;37:877-82.

30. Ostergaard M, Gideon P, Henriksen O, Lorenzen I Synovial volume - a marker of disease severity in rheumatoid arthritis? Quantification by MRI. Scand J Rheumatol 1994;23:197-202.

31. Weishaupt D, Schweitzer ME, Rawool NM, et al. Indirect MR arthrography of the knee: effects of low-intensity ultrasound on the diffusion rate of intravenously administered Gd-DTPA in healthy volunteers. Invest Radiol 2001;36:493-9.

32. Schweitzer ME, Natale P, Winalski CS, Culp R. Indirect wrist MR arthrography: the effects of passive motion versus active exercise. Skeletal Radiol 2000;29:10-4.

33. Yagci B, Manisali M, Yilmaz E, et al. Indirect MR arthrography of the shoulder in detection of rotator cuff ruptures. Eur Radiol 2001;11:258-62.

34. Vahlensieck M, Peterfy CG, Wischer T, et al. Indirect MR arthrography: optimization and clinical applications. Radiology 1996;200:249-54.

35. Imhof H, Nobauer-Huhmann IM, Gahleitner A, et al. Pathophysiology and imaging in inflammatory and blastomatous synovial diseases. Skeletal Radiol 2002;31:313-33.

36. Kingzett-Taylor A, Tirman PF, Feller J, et al. Tendinosis and tears of gluteus medius and minimus muscles as a cause of hip pain: MR imaging findings. AJR 1999;173:1123-6. 